\title{
Top-quark and Higgs boson perspectives at heavy-ion colliders
}

\author{
David d'Enterria
}

CERN, EP Department, 1211 Geneva, Switzerland

\begin{abstract}
The perspectives for measuring the top quark and the Higgs boson in nuclear collisions at the LHC and Future Circular Collider (FCC) are summarized. Perturbative QCD calculations at (N)NLO accuracy, including nuclear parton distribution functions, are used to determine their cross sections and visible yields after standard analysis cuts in $\mathrm{PbPb}$ and $\mathrm{pPb}$ collisions at the $\mathrm{LHC}\left(\sqrt{s_{\mathrm{NN}}}=5.5,8.8 \mathrm{TeV}\right)$ and $\mathrm{FCC}\left(\sqrt{s_{\mathrm{NN}}}=39,63 \mathrm{TeV}\right)$. In their "cleanest" decay channels, $\mathrm{t} \overline{\mathrm{t}} \rightarrow \mathrm{b} \overline{\mathrm{b}} 2 \ell 2 v$ and $\mathrm{H} \rightarrow \gamma \gamma ; 4 \ell$, about $10^{3}\left(10^{5}\right)$ top-quark and $10\left(10^{3}\right)$ Higgs-boson events are expected at the LHC (FCC) for their total nominal integrated luminosities. Whereas the $t \overline{\mathrm{t}}$ observation is clearcut at both colliders, evidence for Higgs production, perfectly possible at the FCC, requires integrating $\times 30$ more luminosities at the LHC.
\end{abstract}

Keywords: Top quark, Higgs boson, heavy-ions, LHC, FCC

\section{Introduction}

The top quark and the Higgs boson (together with the $\tau$ lepton) are the only elementary Standard Model (SM) particles that remain unobserved so far in nuclear collisions. Their production cross sections in hadronic collisions are dominated by gluon-gluon fusion processes $(\mathrm{g} g \rightarrow \mathrm{t} \overline{\mathrm{t}}+\mathrm{X} ; \mathrm{H}+\mathrm{X})$, computable today at the highest degree of theoretical accuracy: NNLO+NNLL for the top quark [1], and $\mathrm{N}^{3} \mathrm{LO}$ for the $\mathrm{H}$ boson [2]. The study of their yield modifications in heavy-ion compared to pp collisions would provide novel extremely well calibrated probes of the initial and final pA and AA states. Both elementary particles, the heaviest known, have very different decay channels and lifetimes, and can thereby be used to uniquely probe various aspects of strongly-interacting matter in nuclear collisions. On the one hand, the top-quark decays very rapidly before hadronizing $\left(\tau_{0}=\hbar / \Gamma_{\mathrm{t}} \approx 0.1 \mathrm{fm} / \mathrm{c}\right.$, much shorter than typical $O(1 \mathrm{fm} / \mathrm{c})$ QGP formation times) into $\mathrm{t} \rightarrow \mathrm{Wb}$ with $\sim 100 \%$ branching ratio, with the $\mathrm{W}$ themselves decaying either leptonically $(\mathrm{t} \rightarrow \mathrm{Wb} \rightarrow \ell v, \mathrm{~b}, 1 / 3$ of the times) or hadronically ( $\mathrm{t} \rightarrow \mathrm{Wb} \rightarrow \mathrm{q} \overline{\mathrm{q}} \mathrm{b}, 2 / 3$ of the times). The kinematical distributions of the charged leptons $(\ell=\mathrm{e}, \mu$ unaffected by any final-state interac- tions) from $\mathrm{t} \overline{\mathrm{t}} \rightarrow \mathrm{b} \overline{\mathrm{b}} 2 \ell 2 v \mathrm{t} \overline{\mathrm{t}}$ decays provide accurate information [3] on the underlying nuclear gluon distribution function in the unexplored high- $x$ region where "antishadowing" and "EMC" effects are supposed to modify its shape compared to the free proton case [4]. On the other hand, the Higgs boson has a lifetime of $\tau_{0} \approx 50 \mathrm{fm} / \mathrm{c}$ and, once produced, traverses the produced medium and scatters with the surrounding partons, resulting in a potential depletion of its yields compared to the pp case [5]. The amount of Higgs boson suppression can be thereby used to accurately determine the final-state density of the produced QGP. The perspectives of t-quark [3] and Higgs boson [6] measurements in nuclear collisions at current and future colliders are summarized here.

The theoretical cross sections and yields in $\mathrm{pPb}$ and $\mathrm{PbPb}$ are obtained with MCFM at NLO (v.6.7) for top-quarks [7], and at NNLO (v.8.0) for the Higgs boson [8], using CT10 proton PDFs [9] and EPS09 nPDFs (including its 30 eigenvalues sets) for the $\mathrm{Pb}$ ion [4]. The following MCFM processes are run: 141 for $\mathrm{t} \overline{\mathrm{t}}, 161,166,171,176,181,186$ for single-top in the $t$-, $s$-channels and associated with W [3]; and 119,116 for gluon-fusion $\mathrm{H} \rightarrow \gamma \gamma, 4 \ell$ (plus 285,90 

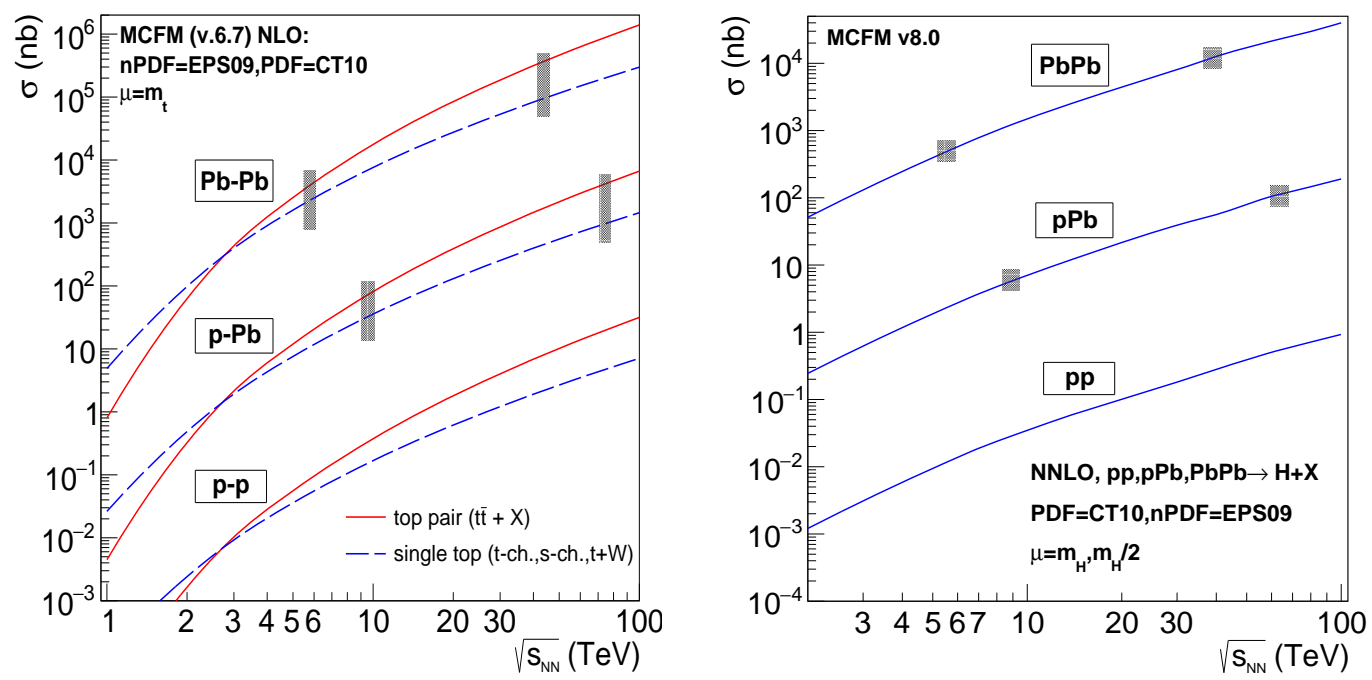

Figure 1: Total production cross sections for top-pair and single-top at NLO (left) [3] and for the Higgs boson at NNLO (right) [6] in pp, pPb and $\mathrm{PbPb}$ collisions as a function of $\sqrt{s_{\mathrm{NN}}}$ (the shaded boxes indicate the nominal LHC and FCC energies).

Table 1: Cross sections, and expected number of counts after standard acceptance and efficiency cuts, for $\mathrm{t} \overline{\mathrm{t}}$ and single-top (s-,t- and $\mathrm{t} \mathrm{W}$ channels combined) at NLO (total, and in their respective leptonic decays) [3] and Higgs boson (total, and di- $\gamma$ and 4-lepton channels, at NNLO) [6] in pPb and $\mathrm{PbPb}$ collisions at $\mathrm{LHC}$ and FCC. Maximum theoretical (scale and PDF) uncertainties (not quoted) are around $10 \%$.

\begin{tabular}{lcc|cc|cc|ccc}
\hline \hline System & $\begin{array}{c}\sqrt{s_{\mathrm{NN}}} \\
(\mathrm{TeV})\end{array}$ & $\mathcal{L}_{\text {int }}$ & $\mathrm{t} \overline{\mathrm{t}}$ & $\mathrm{t} \overline{\mathrm{t}} \rightarrow \mathrm{b} \overline{\mathrm{b}} \ell \ell \nu \nu$ & single-t & $\mathrm{tW} \rightarrow \mathrm{b} \ell \ell \nu \nu$ & $\mathrm{H}$ & $\mathrm{H} \rightarrow \gamma \gamma$ & $\mathrm{H} \rightarrow \mathrm{ZZ}^{*}(4 \ell)$ \\
yields & $\sigma_{\text {tot }}$ & yields & $\sigma_{\text {tot }}$ & yields & yields \\
\hline $\mathrm{PbPb}$ & 5.5 & $10 \mathrm{nb}^{-1}$ & $3.4 \mu \mathrm{b}$ & 450 & $2.0 \mu \mathrm{b}$ & 30 & $500 \mathrm{nb}$ & 6 & 0.3 \\
$\mathrm{pPb}$ & 8.8 & $1 \mathrm{pb}^{-1}$ & $59 \mathrm{nb}$ & 750 & $27 \mathrm{nb}$ & 50 & $6.0 \mathrm{nb}$ & 7 & 0.4 \\
\hline $\mathrm{PbPb}$ & 39 & $33 \mathrm{nb}^{-1}$ & $300 \mu \mathrm{b}$ & $1.5 \times 10^{5}$ & $80 \mu \mathrm{b}$ & 8000 & $11.5 \mu \mathrm{b}$ & 450 & 25 \\
$\mathrm{pPb}$ & 63 & $8 \mathrm{pb}^{-1}$ & $3.2 \mu \mathrm{b}$ & $4 \times 10^{5}$ & $775 \mathrm{nb}$ & $2.1 \times 10^{4}$ & $115 \mathrm{nb}$ & 950 & 50 \\
\hline \hline
\end{tabular}

for the corresponding $\gamma \gamma, 4 \ell$ backgrounds), as well as $91,101,215,540$ corresponding to the rest of Higgs production channels (vector-boson-fusion, and associated with $\mathrm{W}, \mathrm{Z}$ and top) to obtain the total $\mathrm{H}$ cross sections [6]. All numerical results have been obtained with the latest SM parameters [10], and fixing the default renormalization and factorization scales at $\mu_{\mathrm{F}}=\mu_{\mathrm{R}}=$ $\mathrm{m}_{\mathrm{t}}$ for $\mathrm{t} \overline{\mathrm{t}}$ and single-top, $\mu_{\mathrm{F}}=\mu_{\mathrm{R}}=\mathrm{p}_{\mathrm{T} \text {,min:b-jet }}=50 \mathrm{GeV}$ for $\mathrm{tW}$, and $\mu_{\mathrm{F}}=\mu_{\mathrm{R}}=\mathrm{m}_{\mathrm{H}} / 2$ for Higgs. These calculations reproduce very well the top and Higgs cross sections measured in pp collisions at $\sqrt{s}=7,8,13 \mathrm{TeV}$ at the LHC. The collision energy dependence of the total top-quark and Higgs boson cross sections are shown in Fig. 1. The cross sections increase by a factor of $\times 55-$ 90 for $t \bar{t}$ and $\times 20$ for the Higgs boson between LHC and FCC energies. The cross sections at the nominal
LHC and FCC energies are listed in Table 11. Compared to the corresponding pp results at each c.m. energy, antishadowing nPDF modifications increase the total top-quark yields by $2-8 \%$, whereas the Higgs cross sections are just slightly enhanced (depleted) by 3\% at the LHC (FCC). The PDF uncertainties, obtained adding in quadrature the CT10 and EPS09 uncertainties, are around (below) $10 \%$ for the top (Higgs) case. The $O(5-10 \%)$ theoretical $\mu_{F}, \mu_{R}$ scale uncertainties, not quoted either, cancel out in the ratios of $(\mathrm{pPb}, \mathrm{PbPb}) /(\mathrm{pp})$ cross sections at the same c.m. energy.

\section{Top quark measurement}

Top quarks decay almost exclusively to a b quark and a $\mathrm{W}$ boson and, in a heavy-ion environment, it is the $\mathrm{W}$ leptonic decays that can be best resolved 

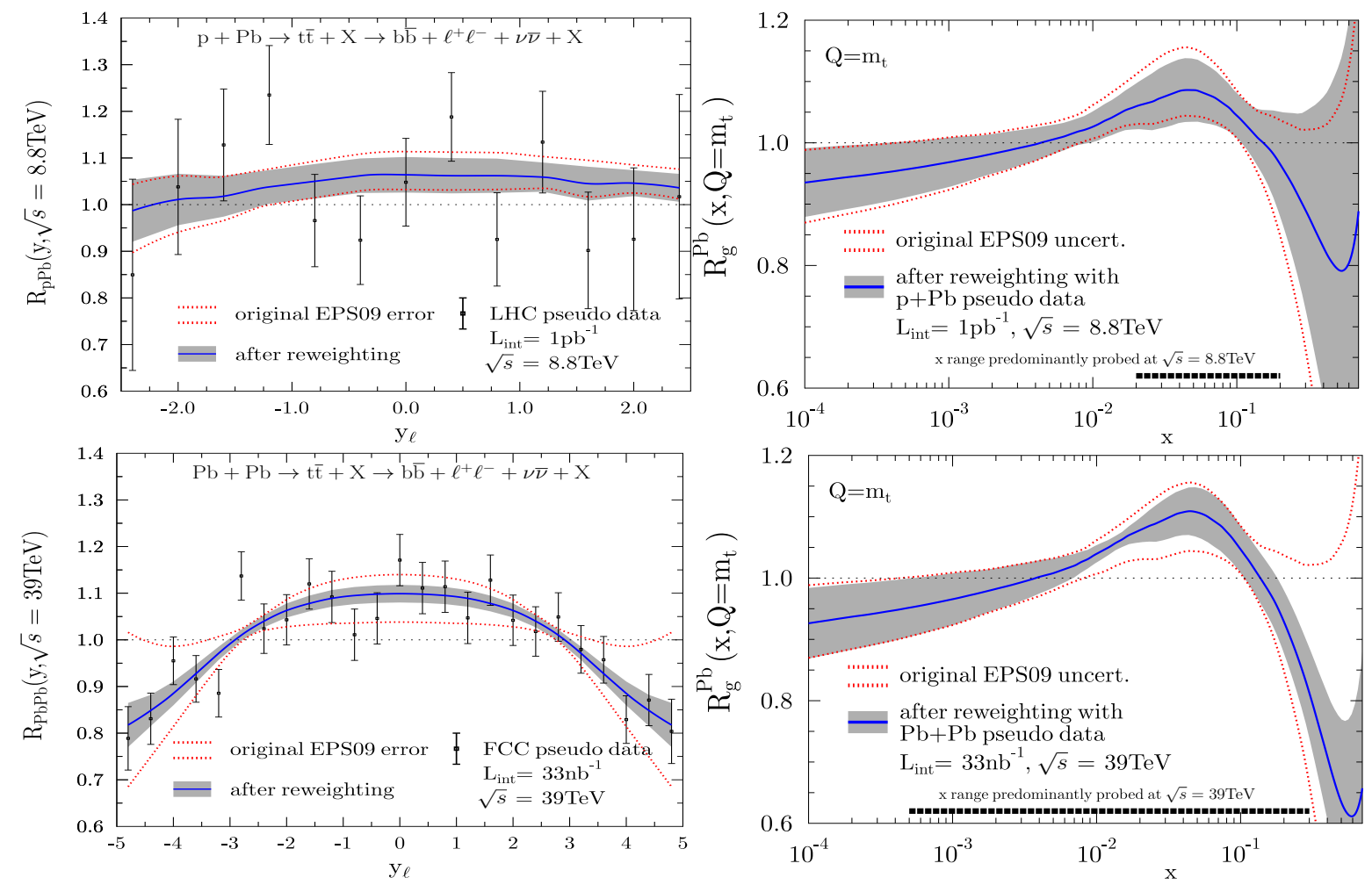

Figure 2: Left: Pseudodata for nuclear modification factors as a function of rapidity of charged leptons produced in t $\overline{\mathrm{t}}$ decays at the $\mathrm{LHC}$ ( $\mathrm{pPb}$, top) [3] and FCC (PbPb, bottom) [11]. Right: Original EPS09 gluon nuclear modification at $Q=m_{\text {top }}$ (red dashed curves) and estimated improvements obtained by Hessian reweighting (grey area) using the $\mathrm{LHC}(\mathrm{pPb}$, top) [3] and $\mathrm{FCC}(\mathrm{PbPb}$, bottom) [11] pseudodata.

from the backgrounds. The estimated measurable yields using realistic luminosities and analysis cuts (b-jets: anti- $k_{\mathrm{T}}$ algorithm with $R=0.5, p_{\mathrm{T}}>30 \mathrm{GeV} / \mathrm{c}$, $|\eta|<3,5$ (LHC, FCC), 50\% b-tagging efficiency; charged leptons: $R_{\text {isol }}=0.3, p_{\mathrm{T}}>20 \mathrm{GeV} / \mathrm{c},|\eta|<$ 3, 5 (LHC, FCC), $\mathrm{m}_{\ell \ell}>20 \mathrm{GeV},\left|\mathrm{m}_{\ell \ell}-\mathrm{m}_{\mathrm{Z}}\right|>15 \mathrm{GeV}$; neutrinos: missing transverse energy $\left.H_{\mathrm{T}}>40 \mathrm{GeV}\right)$ are listed in Table 1 . After branching ratios $(\mathrm{BR} \approx 5 \%$ for $\mathrm{t} \overline{\mathrm{t}}$, $22 \%$ for single-t) and acceptance and efficiency losses (40-50\% for $\mathrm{t} \overline{\mathrm{t}}, 20-30 \%$ for single-t), we expect about 500 and 800 leptonic top-quark events in $\mathrm{PbPb}$ and $\mathrm{pPb}$ collisions at the LHC (with controllable backgrounds in the $t \overline{\mathrm{t}}$ case). At FCC, the higher c.m. energy and luminosities will yield very large data samples with 150-and 400-thousand pairs in $\mathrm{PbPb}$ and $\mathrm{pPb}$. The resulting nuclear modification ratios of $\mathrm{t} \overline{\mathrm{t}}$ decay leptons yields as a function of rapidity, $R_{\mathrm{pPb}}\left(y_{\ell}\right)=d \sigma_{\mathrm{pPb}}\left(y_{\ell}\right) /\left(A d \sigma_{\mathrm{pp}}\left(y_{\ell}\right)\right)$ and $R_{\mathrm{PbPb}}\left(y_{\ell}\right)=d \sigma_{\mathrm{PbPb}}\left(y_{\ell}\right) /\left(A^{2} d \sigma_{\mathrm{pp}}\left(y_{\ell}\right)\right)$, are shown in Fig. 2 (left) for $\mathrm{pPb}$ at the LHC (top) and $\mathrm{PbPb}$ at the FCC (bottom). The effect of antishadowing (shadowing) in the nPDF results in small enhancements (deficits) at central (forward) rapidities $y_{\ell} \approx 0$ ( $\left.\left|y_{\ell}\right| \gtrsim 3\right)$. The impact that these pseudodata would have in an
EPS09 global fit of the nuclear PDFs is quantified via the Hessian reweighting technique [12]. The expected improvements of the gluon PDF nuclear modification factor $R_{\mathrm{g}}^{\mathrm{Pb}}\left(x, Q^{2}\right)=g^{\mathrm{Pb}}\left(x, Q^{2}\right) / g^{\mathrm{p}}\left(x, Q^{2}\right)$ at $Q^{2}=m_{\text {top }}^{2}$ are shown in Fig. 2(right). The uncertainties on the nuclear gluon PDF are reduced by more than $10 \%(50 \%)$ at the LHC (FCC) mostly in the antishadowing and EMC $x$ regions [3, 11].

\section{Higgs boson measurement}

The discovery of the Higgs boson in pp collisions at the LHC was carried out in the clean diphoton and four-lepton channels with very low branching ratios but small and/or controllable backgrounds [13, 14]. A first measurement in heavy-ion collisions will certainly exploit the same final states. Table 1 lists the estimated measurable yields for nominal luminosities after accounting for typical ATLAS/CMS analysis cuts (photons: $p_{\mathrm{T}}>30,40 \mathrm{GeV} / \mathrm{c},|\eta|<2.5,5$ (LHC, FCC), $R_{\text {isol }}=0.3$; charged leptons: $p_{\mathrm{T}}>20,15,10,10 \mathrm{GeV} / \mathrm{c}$, $\left.|\eta|<2.5,5(\mathrm{LHC}, \mathrm{FCC}), R_{\text {isol }}=0.3\right)$. After branching ratios $(\mathrm{BR}=0.23 \%$ for $\gamma \gamma, 0.012 \%$ for $4 \ell)$ and 

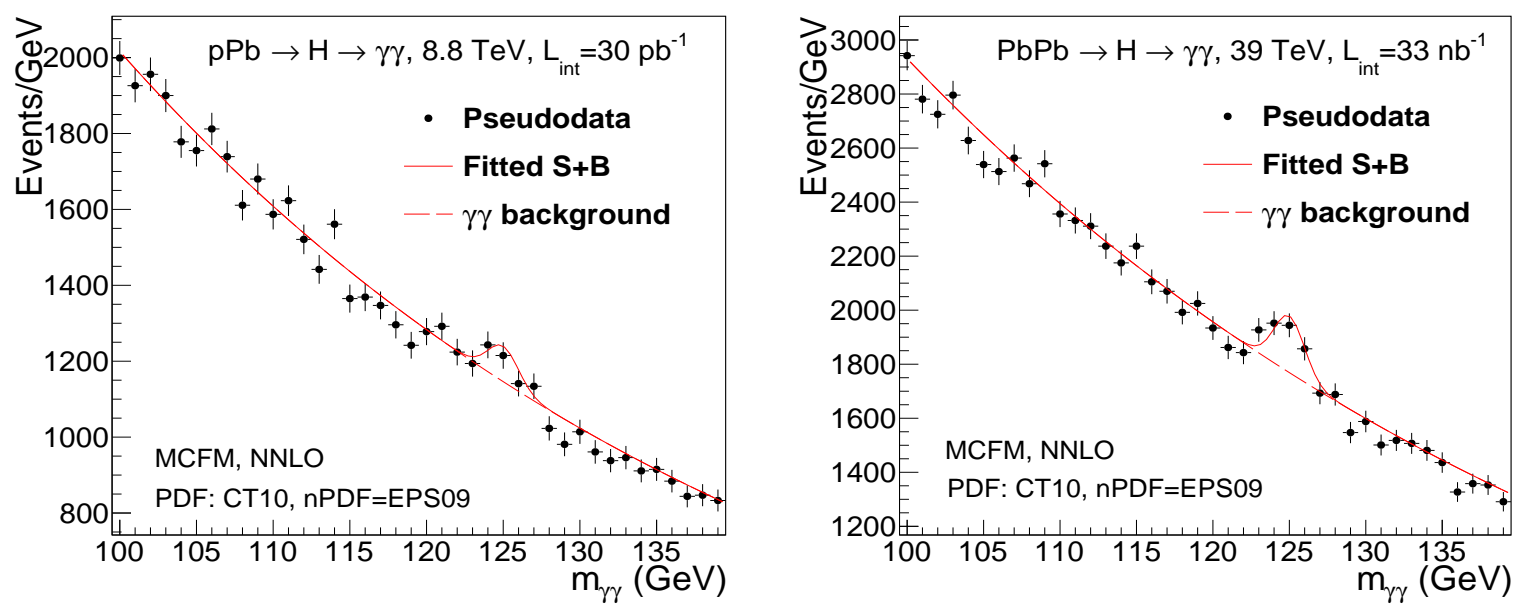

Figure 3: Expected invariant mass diphoton distributions in $\mathrm{pPb}$ at $\sqrt{s_{\mathrm{NN}}}=8.8 \mathrm{TeV}$ (with enhanced LHC integrated luminosities, $\mathcal{L}_{\text {int }}=30 \mathrm{pb}^{-1}$, left) and in $\mathrm{PbPb}$ at $\sqrt{s_{\mathrm{NN}}}=39 \mathrm{TeV}$ (nominal FCC luminosities, right) for a Higgs boson signal injected on top of the $\gamma \gamma$ backgrounds $[6]$.

signal losses from acceptance and efficiency (45-60\% for diphotons, $50 \%$ for 4-leptons), we expect about 10 (1 000) Higgs bosons in $\mathrm{PbPb}$ and $\mathrm{pPb}$ collisions at the LHC (FCC), on top of the corresponding $\gamma \gamma$ and $4 \ell$ nonresonant backgrounds [6]. In the $\gamma \gamma$ case, the backgrounds include the irreducible QCD diphoton continuum plus $30 \%$ of events coming from misidentified $\gamma$ jet and jet-jet processes. For the nominal LHC luminosities, the significance of the diphoton signal (S) over the background (B), computed via $S / \sqrt{B}$ at the Higgs peak, is $0.5,0.6 \sigma$ in $\mathrm{PbPb}$ and $\mathrm{pPb}$ collisions, and thus one needs a factor of $\times 30-40$ larger integrated luminosities to reach $3 \sigma$ evidence (which would be further enhanced by including the $\mathrm{H} \rightarrow 4 \ell$ results). Such a large $\mathcal{L}_{\text {int }}$ increase would require, first, running one fullyear (instead of the nominal heavy-ion month) plus increasing the instantaneous luminosity by a factor of 3-4. Whereas the feasibility of such a setup seems difficult in the $\mathrm{PbPb}$ case, it's not unrealistic in the $\mathrm{pPb}$ mode during the high-luminosity LHC phase (HL-LHC). Figure 3 shows the expected diphoton invariant mass distributions for $\mathrm{pPb}$ at the $\mathrm{LHC}$ (with $\mathcal{L}_{\text {int }}=30 \mathrm{pb}^{-1}$, left) and in $\mathrm{PbPb}$ collisions at the FCC. The significance of the $\mathrm{H}(\gamma \gamma)$ peaks are $3 \sigma$ and $5.5 \sigma$ respectively.

Acknowledgments- Discussions and/or common work with A. David, C. Loizides, and H. Paukkunen are gratefully acknowledged.

\section{References}

[1] M. Czakon, P. Fiedler, A. Mitov, Total Top-Quark PairProduction Cross Section at Hadron Colliders Through $O\left(\alpha_{s}^{4}\right)$,
Phys. Rev. Lett. 110 (2013) 252004. arXiv: 1303.6254 doi : 10.1103/PhysRevLett.110.252004

[2] C. Anastasiou, C. Duhr, F. Dulat, F. Herzog, B. Mistlberger, Higgs Boson Gluon-Fusion Production in QCD at Three Loops, Phys. Rev. Lett. 114 (2015) 212001. arXiv:1503.06056 doi:10.1103/PhysRevLett.114.212001

[3] D. d'Enterria, K. Krajczr, H. Paukkunen, Top-quark production in proton nucleus and nucleus nucleus collisions at LHC energies and beyond, Phys. Lett. B746 (2015) 64-72. arXiv: 1501.05879 doi:10.1016/j.physletb. 2015.04.044

[4] K. J. Eskola, H. Paukkunen, C. A. Salgado, EPS09: A New Generation of NLO Nuclear PDFs, JHEP 04 (2009) 065. arXiv: 0902.4154 doi:10.1088/1126-6708/2009/04/065

[5] D. d'Enterria, C. Loizides, to be submitted.

[6] D. d'Enterria, to be submitted.

[7] J. M. Campbell, R. K. Ellis, MCFM for the Tevatron and the LHC, Nucl. Phys. Proc. Suppl. 205-206 (2010) 10-15. arXiv: 1007.3492 doi:10.1016/j.nuclphysbps.2010.08.011

[8] R. Boughezal, J. M. Campbell, R. K. Ellis, C. Focke, W. Giele, X. Liu, F. Petriello, C. Williams, Color Singlet Production at NNLO in MCFM, Eur. Phys. J. C77 (1) (2017) 7. arXiv: 1605. 08011 doi:10.1140/epjc/s10052-016-4558-y

[9] H.-L. Lai, M. Guzzi, J. Huston, Z. Li, P. M. Nadolsky, J. Pumplin, C. P. Yuan, New parton distributions for collider physics, Phys. Rev. D82 (2010) 074024. arXiv:1007.2241 doi:10.1103/PhysRevD.82.074024

[10] Review of Particle Physics, Chin. Phys. C40 (10) (2016) 100001. doi: $10.1088 / 1674-1137 / 40 / 10 / 100001$

[11] A. Dainese, et al., Heavy ions at the Future Circular ColliderarXiv: 1605.01389

[12] H. Paukkunen, P. Zurita, PDF reweighting in the Hessian matrix approach, JHEP 12 (2014) 100. arXiv: 1402.6623 doi:10. 1007/JHEP12(2014) 100

[13] G. Aad, et al., Observation of a new particle in the search for the Standard Model Higgs boson with the ATLAS detector at the LHC, Phys. Lett. B716 (2012) 1-29. arXiv:1207.7214. doi:10.1016/j.physletb.2012.08.020

[14] S. Chatrchyan, et al., Observation of a new boson at $125 \mathrm{GeV}$ with the CMS experiment at the LHC, Phys. Lett. B716 (2012) 30-61. arXiv:1207.7235 doi:10.1016/j.physletb. 2012.08.021 